\title{
Equations in roots of unity
}

\author{
by \\ Hans Peter Schlickewei (Ulm)
}

1. Introduction. Suppose that $n \geq 1$ and that $a_{1}, \ldots, a_{n}$ are nonzero complex numbers. We study equations

$$
a_{1} \xi_{1}+\ldots+a_{n} \xi_{n}=1
$$

to be solved in roots of unity $\xi_{i}$. We call a solution $\xi=\left(\xi_{1}, \ldots, \xi_{n}\right)$ of $(1.1)$ nondegenerate if $\sum_{i \in I} a_{i} \xi_{i} \neq 0$ for each nonempty subset $I$ of $\{1, \ldots, n\}$. Write $\nu\left(a_{1}, \ldots, a_{n}\right)$ for the number of nondegenerate solutions $\xi$ of (1.1), whose components are roots of unity. Equations (1.1) have been first studied by H. B. Mann [2]. His result implies that for $a_{1}, \ldots, a_{n} \in \mathbb{Q}$ we have

$$
\nu\left(a_{1}, \ldots, a_{n}\right) \leq e^{c_{1} n^{2}},
$$

where $c_{1}$ is a positive absolute constant. This was improved by J. H. Conway and A. J. Jones [1]. They proved that for $a_{1}, \ldots, a_{n} \in \mathbb{Q}$ we get

$$
\nu\left(a_{1}, \ldots, a_{n}\right)=O_{c}\left(\exp \left(c n^{3 / 2}(\log n)^{1 / 2}\right)\right)
$$

for any $c>1$. In the case when $a_{1}, \ldots, a_{n}$ lie in a number field $K$ of degree $d$, A. Schinzel [4] has shown that

$$
\nu\left(a_{1}, \ldots, a_{n}\right) \leq c_{2}(n, d)
$$

for some function $c_{2}$, which depends only upon $n$ and $d$. U. Zannier [7] has improved upon Schinzel's result and also determined $c_{2}$ explicitly.

In the current paper we address the problem to derive a bound for $\nu\left(a_{1}, \ldots, a_{n}\right)$ for arbitrary complex numbers $a_{i}$. We prove that in fact

$$
\nu\left(a_{1}, \ldots, a_{n}\right) \leq c_{3}(n),
$$

i.e., we derive a uniform bound that depends only upon $n$.

Write $E$ for the group of roots of unity. Given a point $\boldsymbol{w}=\left(w_{1}, \ldots, w_{n}\right)$ in $E^{n}$ and a natural number $m$ we write $A(\boldsymbol{w}, m)$ for the set of points $\boldsymbol{v}=\left(v_{1}, \ldots, v_{n}\right) \in E^{n}$ with components of the shape $v_{i}=\eta_{i} w_{i}$, where $\eta_{i}$ is an $m$ th root of unity. We prove 
THEOREM. There exist points $\boldsymbol{w}_{1}, \ldots, \boldsymbol{w}_{t} \in E^{n}$ with

$$
t \leq 2^{2(n+1) !}
$$

and there exist prime numbers

$$
p_{1}<p_{2}<\ldots<p_{s} \leq(n+1) !
$$

with the following property: Any nondegenerate solution $\xi=\left(\xi_{1}, \ldots, \xi_{n}\right)$ of (1.1) in roots of unity is contained in the union

$$
\bigcup_{\tau=1}^{t} A\left(\boldsymbol{w}_{\tau}, p_{1} \ldots p_{s}\right) \text {. }
$$

Moreover, we have for any nonzero complex numbers $a_{1}, \ldots, a_{n}$

$$
\nu\left(a_{1}, \ldots, a_{n}\right) \leq 2^{4(n+1) !} .
$$

We remark at this point that the results of [1], [2], [4] and [7] for rational or algebraic coefficients $a_{i}$ respectively are constructive, i.e., in principle the proofs provide us with algorithms to determine explicitly the solutions $\xi$ of (1.1). This is no longer the case with our Theorem. In fact, it is not clear how our method of proof could give us an effective procedure to construct the points $\boldsymbol{w}_{1}, \ldots, \boldsymbol{w}_{t}$.

In a subsequent paper [5] we will apply our result to estimate the number of solutions of linear equations over division groups of finitely generated subgroups $G$ of the multiplicative group $\mathbb{C}^{*}$. In that wider setting the current Theorem establishes the result of [5] for groups $G$ of rank 0 .

2. Rational coefficients. Suppose $m \geq 2$. Let $b_{1}, \ldots, b_{m}$ be nonzero integers and consider the relation

$$
\sum_{i=1}^{m} b_{i} \xi_{i}=0
$$

where the $\xi_{i}$ are roots of unity. We say that a solution $\xi=\left(\xi_{1}, \ldots, \xi_{m}\right)$ of (2.1) is nondegenerate if for each nonempty proper subset $I$ of $\{1, \ldots, m\}$ we have

$$
\sum_{i \in I} b_{i} \xi_{i} \neq 0
$$

LEMma 2.1. There exist distinct primes $p_{1}, \ldots, p_{u} \leq m$ such that any nondegenerate solution $\xi=\left(\xi_{1}, \ldots, \xi_{m}\right)$ of $(2.1)$ in roots of unity is of the shape

$$
\xi_{i}=\xi \eta_{i},
$$

where the $\eta_{i}$ are $p_{1} \ldots p_{u}$-th roots of unity (and where $\xi$ is a suitable root of unity). 
This is Theorem 1 of H. B. Mann [2].

Lemma 2.2. Let the hypotheses be the same as in Lemma 2.1. Then the primes $p_{1}, \ldots, p_{u}$ satisfy

$$
\sum_{i=1}^{u}\left(p_{i}-2\right) \leq m-2 .
$$

This is Theorem 5 of Conway and Jones [1].

3. Multilinear maps. Let $\mathbb{C}^{N}$ be the vector space of $N$-tuples $\left(x_{1}, \ldots, x_{N}\right)$. Let $\mathfrak{S}_{N}$ be the group of permutations of $\{1, \ldots, N\}$, so that $\left|\mathfrak{S}_{N}\right|=N$ !. Let $V$ be the space of vectors with components $z_{\sigma}\left(\sigma \in \mathfrak{S}_{N}\right)$, so that $\operatorname{dim} V=N$ !. We introduce the map from $\mathbb{C}^{N} \times \ldots \times \mathbb{C}^{N}$ (with $N$ factors) into $V$ given by

$$
\left(\boldsymbol{x}_{1}, \ldots, \boldsymbol{x}_{N}\right) \mapsto \boldsymbol{z}\left(\boldsymbol{x}_{1}, \ldots, \boldsymbol{x}_{N}\right) .
$$

Here $\boldsymbol{z}$ has components

$$
z_{\sigma}=(\operatorname{sign} \sigma) x_{1, \sigma(1)} \ldots x_{N, \sigma(N)},
$$

where $\boldsymbol{x}_{i}=\left(x_{i 1}, \ldots, x_{i N}\right)$ and sign $\sigma= \pm 1$ according as $\sigma$ is an even or odd permutation. It is clear that $\boldsymbol{z}$ is linear in each $\boldsymbol{x}_{i}$ and we have

$$
\sum_{\sigma \in \mathfrak{S}_{N}} z_{\sigma}\left(\boldsymbol{x}_{1}, \ldots, \boldsymbol{x}_{N}\right)=\operatorname{det}\left(\boldsymbol{x}_{1}, \ldots, \boldsymbol{x}_{N}\right) .
$$

Suppose we are given a hyperplane $U$ of $\mathbb{C}^{N}$ defined by the equation

$$
\sum_{i=1}^{N} c_{i} x_{i}=0 .
$$

Assume that the coefficients in (3.3) satisfy

$$
c_{1} \ldots c_{N} \neq 0 \text {. }
$$

Given vectors $\boldsymbol{x}_{1}, \ldots, \boldsymbol{x}_{N} \in U$, we obviously get

$$
\sum_{\sigma \in \mathfrak{S}_{N}} z_{\sigma}\left(\boldsymbol{x}_{1}, \ldots, \boldsymbol{x}_{N}\right)=0 .
$$

Lemma 3.1. Let $d_{\sigma}\left(\sigma \in \mathfrak{S}_{N}\right)$ be complex numbers such that

$$
\sum_{\sigma \in \mathfrak{S}_{N}} d_{\sigma} z_{\sigma}\left(\boldsymbol{x}_{1}, \ldots, \boldsymbol{x}_{N}\right)=0
$$

for each tuple of points $\left(\boldsymbol{x}_{1}, \ldots, \boldsymbol{x}_{N}\right)$ with $\boldsymbol{x}_{i} \in U(1 \leq i \leq N)$. Then $\left(d_{\sigma}\right)_{\sigma \in \mathfrak{S}_{N}}$ is proportional to $(1, \ldots, 1)$, i.e., (3.6) is a consequence of $(3.5)$.

This is Lemma 4 of [6]. 
Write $\mathfrak{M}$ for the set of proper nonempty subsets $\mathfrak{R}$ of $\mathfrak{S}_{N}$. Given $\mathfrak{R} \in \mathfrak{M}$ we define the multilinear form $F_{\mathfrak{R}}$ in vectors $\boldsymbol{x}_{1}, \ldots, \boldsymbol{x}_{N} \in U$, by

$$
F_{\mathfrak{R}}\left(\boldsymbol{x}_{1}, \ldots, \boldsymbol{x}_{N}\right)=\sum_{\sigma \in \mathfrak{R}} z_{\sigma}\left(\boldsymbol{x}_{1}, \ldots, \boldsymbol{x}_{N}\right) .
$$

Lemma 3.1 implies that $F_{\mathfrak{R}}$ does not vanish identically on $U \times \ldots \times U$ ( $N$ factors). We may conclude that the set of points $\boldsymbol{y} \in U$ such that $F_{\mathfrak{R}}\left(\boldsymbol{y}, \boldsymbol{x}_{2}, \ldots, \boldsymbol{x}_{N}\right)$ vanishes identically in $\boldsymbol{x}_{2}, \ldots, \boldsymbol{x}_{N} \in U$ is a proper subspace of $U$. We denote this subspace by $U_{1 \Re}$. We may perform this procedure for every $\mathfrak{R} \in \mathfrak{M}$. Now pick an element $\boldsymbol{y}_{1} \in U \backslash \bigcup_{\mathfrak{R} \in \mathfrak{M}} U_{1 \mathfrak{R}}$. Consider the set of elements $\boldsymbol{y} \in U$ such that $F_{\mathfrak{R}}\left(\boldsymbol{y}_{1} ; \boldsymbol{y}, \boldsymbol{x}_{3}, \ldots, \boldsymbol{x}_{N}\right)$ vanishes identically as $\boldsymbol{x}_{3}, \ldots, \boldsymbol{x}_{N}$ run through $U$. By our choice of $\boldsymbol{y}_{1}$, this condition defines for each $\mathfrak{R} \in \mathfrak{M}$ a proper subspace $U_{2 \mathfrak{R}}$ of $U$. Pick $\boldsymbol{y}_{2}$ in $U \backslash \bigcup_{\mathfrak{R} \in \mathfrak{M}} U_{2 \mathfrak{R}}$. Our set of multilinear forms $F_{\mathfrak{R}}(\mathfrak{R} \in \mathfrak{M})$ is symmetric in the following sense: For each $\mathfrak{R} \in \mathfrak{M}$ and for each $\sigma \in \mathfrak{S}_{N}$ there exists $\mathfrak{R}^{\prime} \in \mathfrak{M}$ such that

$$
F_{\mathfrak{R}}\left(\boldsymbol{x}_{\sigma(1)}, \ldots, \boldsymbol{x}_{\sigma(N)}\right)= \pm F_{\mathfrak{R}^{\prime}}\left(\boldsymbol{x}_{1}, \ldots, \boldsymbol{x}_{n}\right) .
$$

It follows that we have

$$
\bigcup_{\mathfrak{R} \in \mathfrak{M}} U_{2 \mathfrak{R}} \supset \bigcup_{\mathfrak{R} \in \mathfrak{M}} U_{1 \mathfrak{R}}
$$

We may continue our construction in an obvious way. Finally, we get points $\boldsymbol{y}_{1}, \ldots, \boldsymbol{y}_{N-1}$ and subspaces $U_{i \mathfrak{R}}$ for $i=1, \ldots, N-1$ and $\mathfrak{R} \in \mathfrak{M}$. Then each equation

$$
F_{\mathfrak{R}}\left(\boldsymbol{y}_{1}, \ldots, \boldsymbol{y}_{N-1}, \boldsymbol{x}\right)=0 \quad \text { in } \boldsymbol{x} \in U
$$

will define a proper subspace $U_{N \Re}$ of $U$ and we have

$$
\bigcup_{\mathfrak{R} \in \mathfrak{M}} U_{N \mathfrak{R}} \supset \bigcup_{\mathfrak{R} \in \mathfrak{M}} U_{N-1, \mathfrak{R}} \supset \ldots \supset \bigcup_{\mathfrak{R} \in \mathfrak{M}} U_{1 \mathfrak{R}} .
$$

Our construction now implies

LEMma 3.2. Suppose the points $\boldsymbol{y}_{1}, \ldots, \boldsymbol{y}_{N-1}$ and the subspaces $U_{N \Re}$ $(\mathfrak{R} \in \mathfrak{M})$ are constructed as above. Then for each point $\boldsymbol{x} \in U \backslash \bigcup_{\mathfrak{R} \in \mathfrak{M}} U_{N \Re}$ we have

$$
\sum_{\sigma \in \mathfrak{S}_{N}} z_{\sigma}\left(\boldsymbol{y}_{1}, \ldots, \boldsymbol{y}_{N-1}, \boldsymbol{x}\right)=0
$$

but no proper nonempty subsum of the left hand side of (3.9) vanishes.

For the proof it suffices to observe that the subsums of (3.9) are just our multilinear forms $F_{\mathfrak{R}}$. But our construction is such that for $\boldsymbol{x} \in U \backslash$ $\bigcup_{\mathfrak{R} \in \mathfrak{M}} U_{N \mathfrak{R}}$ we have $F_{\mathfrak{R}}\left(\boldsymbol{y}_{1}, \ldots, \boldsymbol{y}_{N-1}, \boldsymbol{x}\right) \neq 0$ for each $\mathfrak{R} \in \mathfrak{M}$. 
We finally remark that the number of subspaces $U_{N \mathfrak{R}}(\mathfrak{R} \in \mathfrak{M})$ is bounded by

$$
2^{N !}
$$

4. Linear subspaces. Let $N \geq 2$ and suppose that $c_{1}, \ldots, c_{N}$ are nonzero complex numbers. Consider the subspace $U$ of $\mathbb{C}^{N}$ defined by

$$
c_{1} x_{1}+\ldots+c_{N} x_{N}=0
$$

which was already studied in Section 3 . Write $B$ for the subset of points $\boldsymbol{x}=\left(x_{1}, \ldots, x_{N}\right)$ in $U$ whose components $x_{i}$ are roots of unity. Denote by $E$ the set of all roots of unity and by $E_{m}$ the set of $m$ th roots of unity. If $B \neq \emptyset$, we define for $\boldsymbol{u} \in B$ and for $l \in \mathbb{N}$ the set $B(\boldsymbol{u}, l)$ by

$$
B(\boldsymbol{u}, l)=\left\{\left(\zeta \zeta_{1} u_{1}, \ldots, \zeta \zeta_{N} u_{N}\right) \mid \zeta \in E, \zeta_{i} \in E_{l}\right\} .
$$

Lemma 4.1. Suppose that $B \neq \emptyset$. Then there exists $\boldsymbol{u}_{0} \in B$, there are primes $q_{1}<\ldots<q_{a}$ with

$$
\sum_{i=1}^{a}\left(q_{i}-2\right) \leq N !-2
$$

and there are proper linear subspaces $W_{1}, \ldots, W_{t_{1}}$ of $U$ such that the following assertion holds true: The set of solutions $B$ of (4.1) in roots of unity is contained in the union

$$
B\left(\boldsymbol{u}_{0}, q_{1} \ldots q_{a}\right) \cup W_{1} \cup \ldots \cup W_{t_{1}} .
$$

Here we have

$$
t_{1} \leq 2^{N !}
$$

Proof. Recall the definition of the multilinear forms $F_{\mathfrak{R}}\left(\boldsymbol{x}_{1}, \ldots, \boldsymbol{x}_{N}\right)$ in (3.7). The subspaces $U_{1 \mathfrak{R}}(\mathfrak{R} \in \mathfrak{M})$ in Section 3 do not depend on any choice of points. We distinguish two cases: Either $B$ is contained in the union $\bigcup_{\mathfrak{R} \in \mathfrak{M}} U_{1 \mathfrak{R}}$. Then obviously the assertion of the lemma is satisfied with $\left\{W_{1}, \ldots, W_{t_{1}}\right\}=\left\{U_{1 \mathfrak{R}} \mid \mathfrak{R} \in \mathfrak{M}\right\}$. In that case the set $B\left(\boldsymbol{u}_{0}, q_{1} \ldots q_{a}\right)$ will not be needed at all. So any $\boldsymbol{u}_{0} \in B$ and any primes $q_{1}<\ldots<q_{a}$ as in the assertion will do. Otherwise we may pick $\boldsymbol{y}_{1} \in B \backslash \bigcup_{\mathfrak{R} \in \mathfrak{M}} U_{1 \mathfrak{R}}$ and define subspaces $U_{2 \mathfrak{R}}(\mathfrak{R} \in \mathfrak{M})$ with respect to $\boldsymbol{y}_{1}$. Then if $B \subset \bigcup_{\mathfrak{R} \in \mathfrak{M}} U_{2 \mathfrak{R}}$, we may choose $\boldsymbol{u}_{0} \in B, q_{1}<q_{2}<\ldots<q_{a}$ according to the assertion arbitrarily and again the lemma follows. Continuing in this way, there are two alternatives: either the construction ends after step $j$ with $j \leq N-1, B$ will be contained in the union $\bigcup_{\mathfrak{R} \in \mathfrak{M}} U_{j \Re}$ and we are done. Or we may find points $\boldsymbol{y}_{1}, \ldots, \boldsymbol{y}_{N-1} \in B$ and define subspaces $U_{N \mathfrak{R}}(\mathfrak{R} \in \mathfrak{M})$ with respect to these points. 
Then we take $\left\{W_{1}, \ldots, W_{t_{1}}\right\}=\left\{U_{N \mathfrak{R}} \mid \mathfrak{R} \in \mathfrak{M}\right\}$. We now assume that $B \backslash \bigcup_{\mathfrak{R} \in \mathfrak{M}} U_{N \mathfrak{R}} \neq \emptyset$. We may apply Lemma 3.2 and conclude that for each $\boldsymbol{x} \in B \backslash \bigcup_{\mathfrak{R} \in \mathfrak{M}} U_{N \Re}$

$$
\sum_{\sigma \in \mathfrak{S}_{N}} z_{\sigma}\left(\boldsymbol{y}_{1}, \ldots, \boldsymbol{y}_{N-1}, \boldsymbol{x}\right)=0
$$

but no proper nonempty subsum of the left hand side of (4.4) vanishes. However, by definition of $B$, the summands $z_{\sigma}\left(\boldsymbol{y}_{1}, \ldots, \boldsymbol{y}_{N-1}, \boldsymbol{x}\right)$ in (4.4) are roots of unity. So (4.4) is an equation of the type considered in (2.1), and in fact we have nondegenerate solutions $\left(z_{\sigma}\left(\boldsymbol{y}_{1}, \ldots, \boldsymbol{y}_{N-1}, \boldsymbol{x}\right)\right)_{\sigma \in \mathfrak{S}_{N}}$. We may apply Lemma 2.2 with $m=N$ ! and with $b_{1}=\ldots=b_{m}=1$. Now Lemma 2.2 says the following: For any solution $\boldsymbol{x} \in B \backslash \bigcup_{\mathfrak{R} \in \mathfrak{M}} U_{N \mathfrak{R}}$ the point $\left(z_{\sigma}\left(\boldsymbol{y}_{1}, \ldots, \boldsymbol{y}_{N-1}, \boldsymbol{x}\right)\right)_{\sigma \in \mathfrak{S}_{N}}$ is of the shape $\left(\zeta \eta_{\sigma}\right)_{\sigma \in \mathfrak{S}_{N}}$, where $\zeta$ is an arbitrary root of unity and where the $\eta_{\sigma}$ are $q_{1} \ldots q_{a}$-th roots of unity with

$$
\sum_{i=1}^{a}\left(q_{i}-2\right) \leq N !-2 .
$$

We may assume without loss of generality that $2 \in\left\{q_{1}, \ldots, q_{a}\right\}$. Otherwise we enlarge the set by taking $\left\{2, q_{1}, \ldots, q_{a}\right\}$.

Recall that the components $z_{\sigma}$ are the summands in the Laplace expansion of the determinant

$$
\operatorname{det}\left(\boldsymbol{y}_{1}, \ldots, \boldsymbol{y}_{N-1}, \boldsymbol{x}\right)=\left|\begin{array}{ccc}
y_{11} & \ldots & y_{1 N} \\
y_{21} & \ldots & y_{2 N} \\
\ldots \ldots & \ldots & \ldots \\
x_{1} & \ldots & x_{N}
\end{array}\right| .
$$

We claim that for $i=1, \ldots, N$ we can find a root of unity $\zeta_{i}$ of order dividing $q_{1} \ldots q_{a}$ such that

$$
x_{i}=\frac{x_{1}}{y_{11}} y_{1 i} \zeta_{i}
$$

holds true for $i=1, \ldots, N$.

To verify (4.5) consider in the expansion of the determinant the element we get along the main diagonal, i.e., $y_{11} y_{22} \ldots y_{N-1, N-1} x_{N}$. Compare this element with the one where we replace the top left corner by the bottom left corner and the bottom right corner with the top right corner but otherwise we remain on the main diagonal, i.e., consider the element $x_{1} y_{22} \ldots y_{N-1, N-1} y_{1 N}$. Taking quotients we get

$$
\frac{y_{11} x_{N}}{y_{1 N} x_{1}}= \pm \eta_{N}
$$

where $\eta_{N}$ is a root of unity of order dividing $q_{1} \ldots q_{a}$. By our assumption $2 \in$ $\left\{q_{1}, \ldots, q_{a}\right\}$ however, and therefore $-\eta_{N}$ is a root of unity of order dividing $q_{1} \ldots q_{a}$ as well. Thus (4.5) in the case $i=N$ is verified with $\zeta_{N}= \pm \eta_{N}$. 
Interchanging columns in the determinant we get (4.5) in general. Therefore taking $\boldsymbol{u}_{0}=\boldsymbol{y}_{1}$ we may infer that each point $\boldsymbol{x} \in B \backslash \bigcup_{\mathfrak{R} \in \mathfrak{M}} U_{N \mathfrak{R}}$ is of the shape

$$
\left(x_{1}, \ldots, x_{N}\right)=\left(\zeta \zeta_{i} y_{1 i}\right)=\left(\zeta \zeta_{i} u_{0 i}\right) \quad \text { with } \zeta=x_{1} / y_{11}
$$

and the lemma follows.

5. Proof of the Theorem. For $n=1$ equation (1.1) has at most a single solution in $E$ and the Theorem follows trivially.

Now suppose that $n>1$ and that the assertion is proved for all $n^{\prime}<n$. Put $n+1=N$ and write equation (1.1) in homogenized form as

$$
a_{1} x_{1}+\ldots+a_{N-1} x_{N-1}-x_{N}=0
$$

to be solved in roots of unity $x_{i}$.

To prove assertion (1.2) of the Theorem it will suffice to show that there are points $\boldsymbol{u}_{1}, \ldots, \boldsymbol{u}_{t} \in E^{N}$ with

$$
t \leq 2^{2 N !}
$$

such that any nondegenerate solution $\boldsymbol{x}=\left(x_{1}, \ldots, x_{N}\right)$ of $(5.1)$ is contained in the union

$$
\bigcup_{\tau=1}^{t} B\left(\boldsymbol{u}_{\tau}, p_{1} \ldots p_{s}\right)
$$

with $B(\boldsymbol{u}, l)$ as in (4.2). Then clearly the general solution $\boldsymbol{\xi}=\left(\xi_{1}, \ldots, \xi_{n}\right)$ of (1.1) will be of the shape

$$
\xi_{i}=\frac{x_{i}}{x_{N}} \quad(i=1, \ldots, n) .
$$

Thus in the Theorem the sets $A\left(\boldsymbol{w}_{\tau}, p_{1} \ldots p_{s}\right)(\tau=1, \ldots, t)$ will do, where $\boldsymbol{w}_{\tau}=\left(w_{\tau 1}, \ldots, w_{\tau n}\right)$ is defined by

$$
w_{\tau i}=\frac{u_{\tau i}}{u_{\tau N}} \quad(i=1, \ldots, n) .
$$

We may apply Lemma 4.1 to (5.1). Thus, there exist proper linear sub-

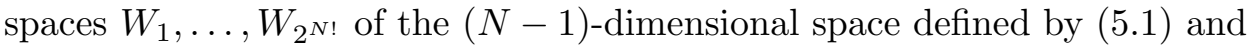
there exists a tuple $\boldsymbol{u}_{0}=\left(u_{01}, \ldots, u_{0 N}\right)$ of roots of unity such that any solution $\boldsymbol{x}=\left(x_{1}, \ldots, x_{N}\right)$ of (5.1) is contained in the union

$$
B\left(\boldsymbol{u}_{0}, q_{1} \ldots q_{a}\right) \cup W_{1} \cup \ldots \cup W_{2^{N !}} .
$$

Here

$$
\sum_{i=1}^{a}\left(q_{i}-2\right) \leq N !-2 .
$$


Consider a typical subspace, say $W$. It may be defined by an equation

$$
b_{1} x_{1}+\ldots+b_{N-1} x_{N-1}=0,
$$

where not all $b_{i}$ are equal to zero.

Let $I$ be a nonempty subset of $\{1, \ldots, N-1\}$ and let $C(I)$ be the subset of solutions of (5.2) satisfying

$$
\sum_{i \in I} b_{i} x_{i}=0
$$

but no proper subsum of the left hand side of (5.3) vanishes. We may apply the induction hypothesis to the solutions of (5.3) lying in $C(I)$.

Consequently, there exist $2^{2|I| !}$ points $\boldsymbol{v}_{j}$ in $|I|$-dimensional space, and with roots of unity as components, such that any nondegenerate solution of (5.3) is contained in the union

$$
\bigcup B\left(\boldsymbol{v}_{j}, r_{1} \ldots r_{b}\right)
$$

Here $r_{1}, \ldots, r_{b}$ are suitable primes satisfying $r_{1}<\ldots<r_{b} \leq|I|$ !.

Let $\boldsymbol{v}=\left(v_{i}\right)_{i \in I}$ be a typical point among the $\boldsymbol{v}_{j}$. Then the elements in $B\left(\boldsymbol{v}, r_{1} \ldots r_{b}\right)$ may be written as $\left(x_{i}\right)_{i \in I}$ with components

$$
x_{i}=x \eta_{i} v_{i} \quad(i \in I),
$$

where $x$ is an arbitrary root of unity and $\eta_{i}$ is a root of unity whose order divides $r_{1} \ldots r_{b}$. We may substitute (5.4) into (5.1) and obtain, writing $a_{N}=$ -1 ,

$$
\left(\sum_{i \in I} a_{i} \eta_{i} v_{i}\right) x+\sum_{i \notin I} a_{i} x_{i}=0
$$

This is an equation in the $N-|I|+1$ variables $x_{i}$ with $i \notin I$ and $x$. As in (5.3) we clearly have $|I| \geq 2$, we may apply the inductive hypothesis again. By the hypothesis of our Theorem, on the left hand side of (5.5) no proper subsum vanishes. Thus by induction we get $2^{2(N-|I|+1) !}$ points $\boldsymbol{y}_{j} \in E^{N-|I|+1}$ and primes $s_{1}<\ldots<s_{c} \leq(N-|I|+1)$ ! such that the solutions $x, x_{i}(i \notin I)$ of (5.5) are contained in the union of the sets $B\left(\boldsymbol{y}_{j}, s_{1} \ldots s_{c}\right)$. We now combine the results we have derived for (5.3) and (5.5).

Given a point $\boldsymbol{v}$ corresponding to (5.3) and a point $\boldsymbol{y}$ corresponding to (5.5) we construct a point $\boldsymbol{u} \in E^{N}$ as follows: Suppose $\boldsymbol{y}$ has the components $y, y_{i}(i \notin I)$. Then we put

$$
u_{i}= \begin{cases}v_{i} y & \text { for } i \in I, \\ y_{i} & \text { for } i \notin I .\end{cases}
$$

So $\boldsymbol{u}=\left(u_{1}, \ldots, u_{N}\right)$ has components which are roots of unity. 
Recall that the subspace $W$ with the set $I$ produces $2^{2|I| !}$ points $\boldsymbol{v}$ and $2^{2(N-|I|+1) !}$ points $\boldsymbol{y}$. So the pair $W, I$ gives rise to no more than

$$
2^{2(|I| !+(N-|I|+1) !)} \quad \text { points } \boldsymbol{u} .
$$

A closer look at (5.3) and (5.5) shows that for $|I|=2$ in (5.3) a single point $\boldsymbol{v}$ and for $|I|=N-1$ in (5.5) a single point $\boldsymbol{y}$ will suffice. In both cases we obtain only $2^{2(N-1) !}$ points $\boldsymbol{u}$. Estimating the total number of points $\boldsymbol{u}$ corresponding to a single subspace $W$ we finally obtain the bound

$$
\begin{aligned}
\left(\left(\begin{array}{c}
N \\
2
\end{array}\right)+\left(\begin{array}{c}
N \\
N-1
\end{array}\right)\right) 2^{2(N-1) !}+\sum_{|I|=3}^{N-2}\left(\begin{array}{l}
N \\
|I|
\end{array}\right) 2^{2(|I| !+(N-|I|+1) !)} & \\
& \leq 2^{2(N-1) !}\left(2^{N}-N\right) .
\end{aligned}
$$

Consequently, each subspace $W$ gives rise to not more than $2^{N !}-1$ points $\boldsymbol{u}$.

Allowing a factor $2^{N !}$ from Lemma 4.1 for the number of subspaces $W$, we finally see that altogether $2^{2 N !}$ points $\boldsymbol{u}$ will suffice for the set of subspaces $W$. This bound also easily takes care of the extra point $\boldsymbol{u}_{0}$ arising from the assertion of Lemma 4.1.

We still have to discuss how the primes $r_{1}, \ldots, r_{b}$ and $s_{1}, \ldots, s_{c}$ from (5.3) and (5.5) and the primes $q_{1}, \ldots, q_{a}$ corresponding to $\boldsymbol{u}_{0}$ fit together. However, we had

$$
\begin{gathered}
q_{1}<\ldots<q_{a}, \quad \sum_{i=1}^{a}\left(q_{i}-2\right) \leq N !-2, \\
r_{1}<\ldots<r_{b} \leq|I| !, \\
s_{1}<\ldots<s_{c} \leq(N-|I|+1) ! .
\end{gathered}
$$

Therefore, we have only primes not exceeding $N$ !. Thus in the assertion of the Theorem it suffices to take the set $\left\{p_{1}, \ldots, p_{s}\right\}$ as the union of the sets $\left\{r_{1}, \ldots, r_{b}\right\},\left\{s_{1}, \ldots, s_{c}\right\}$, the union being taken over all points $\boldsymbol{v}$ and $\boldsymbol{y}$ in our construction together with the primes $\left\{q_{1}, \ldots, q_{a}\right\}$ coming from the extra point $\boldsymbol{u}_{0}$ in Lemma 4.1. Assertion (1.2) follows with $N=n+1$.

As for assertion (1.3), we remark that by Theorem 9 of J. B. Rosser and L. Schoenfeld [3] we have for $x>0$

$$
\sum_{p \leq x} \log p \leq 1.02 x
$$

In the above considerations leading to the proof of (1.2), it is clear that points $\boldsymbol{w}_{\tau}$ coming from a subspace $W_{\tau}$ involve only primes $\leq(N-1)$ !. In view of (5.7) there will be not more than $\exp (1.02(N-1) !)$ possibilities for each component $\xi_{i}$ of a solution $\xi$ in the corresponding set $A\left(\boldsymbol{w}_{\tau}, p_{1} \ldots p_{s}\right)$. Hence $A\left(\boldsymbol{w}_{\tau}, p_{1} \ldots p_{s}\right)$ in that case contains not more than $2^{2(n+1) !}$ solutions $\xi$. 
There remains the exceptional point $\boldsymbol{u}_{0}$ in Lemma 4.1. The corresponding set $B\left(\boldsymbol{u}_{0}, q_{1} \ldots q_{a}\right)$ in view of Lemmata 2.1 and 2.2 has

$$
q_{1}<\ldots<q_{a}, \quad \sum_{i=1}^{a}\left(q_{i}-2\right) \leq N !-2 .
$$

Estimating roughly, again we see that there are not more than $2^{2(n+1) !}$ solutions $\boldsymbol{\xi}$ in the set $A\left(\boldsymbol{w}_{0}, q_{1} \ldots q_{a}\right)$ derived from $B\left(\boldsymbol{u}_{0}, q_{1} \ldots q_{a}\right)$. Allowing the factor $2^{2(n+1) !}$ for the number of sets $A\left(\boldsymbol{w}_{\tau}, p_{1} \ldots p_{s}\right)$ we finally get (1.3).

\section{References}

[1] J. H. Conway and A. J. Jones, Trigonometric diophantine equations (On vanishing sums of roots of unity), Acta Arith. 30 (1976), 229-240.

[2] H. B. Mann, On linears relations between roots of unity, Mathematika 12 (1965), 107-117.

[3] J. B. Rosser and L. Schoenfeld, Approximate formulas for some functions of prime numbers, Illinois J. Math. 6 (1962), 64-94.

[4] A. Schinzel, Reducibility of lacunary polynomials, VIII, Acta Arith. 50 (1988), 91-106.

[5] H. P. Schlickewei, Linear equations over groups of finite rank, to appear.

[6] H. P. Schlickewei and W. M. Schmidt, On polynomial-exponential equations, Math. Ann. 296 (1993), 339-361.

[7] U. Zannier, On the linear independence of roots of unity over finite extensions of $\mathbb{Q}$, Acta Arith. 52 (1989), 171-182.

Abteilung Mathematik II

Universität Ulm

Helmholzstr. 18

D-89069 Ulm, Germany

E-mail: hps@mathematik.uni-ulm.de 